\title{
Medizinische Leitlinien sind wettbewerbsrechtlich nicht justiziabel
}

\section{Zusammenfassung}

\section{Landgericht Köln weist Klage eines Pharmaunternehmens ab}

Das Landgericht Köln hat mit seinem Urteil vom 30.11.2011 die Klage eines Pharmaunternehmens gegen die Träger der Nationalen Versorgungsleitlinien (NVL) zurückgewiesen. Zwar seien die Arbeitsgemeinschaft der Wissenschaftlichen Medizinischen Fachgesellschaften (AWMF), die Bundesärztekammer (BÄK) und die Kassenärztliche Bundesvereinigung (KBV) als Träger und Herausgeber der NVL grundsätzlich für den Inhalt solcher Leitlinien verantwortlich. Äußerungen in Leitlinien zur Anwendung bestimmter Arzneiwirkstoffe oder anderer medizinischer Verfahren könnten grundsätzlich in den eingerichteten und ausgeübten Gewerbebetrieb eines Pharmaunternehmens oder eines anderen Dritten eingreifen. Sie könnten, soweit sie Tatsachenbehauptungen beinhalten, auch geeignet sein, im Sinne einer Kreditgefährdung des § 824 BGB Nachteile für den Erwerb oder das Fortkommen eines gewerblichen Unternehmens herbeizuführen. Bei den hier angegriffenen Äußerungen handele es sich allerdings durchgängig um Bewertungen und Meinungsäußerungen, deren Unterlassung oder Berichtigung das Pharmaunternehmen nicht beanspruchen könne. Wettbewerbsrechtliche Ansprüche bestünden daneben ebenfalls nicht.

\section{Text}

Entscheidend sei im Einzelfall die Abgrenzung, ob es sich bei den angegriffenen Äußerungen um Tatsachenbehauptungen oder um Meinungsäußerungen handele. Während wahre Tatsachenbehauptungen grundsätzlich hinzunehmen seien, seien unwahre Tatsachenbehauptungen grundsätzlich nicht zu dulden. Demgegenüber unterfielen Meinungsäußerungen grundsätzlich dem Schutz von Art. 5 Abs. 1 des Grundgesetzes (GG). Der Unternehmer müsse daher kritische Äußerungen über seine unternehmerischen Leistungen bis zur Grenze der Schmähkritik hinnehmen. Erfolgten die Äußerungen im Rahmen von vergleichenden Warentest oder sonstigen Test und Bewertungen, sei regelmäßig davon auszugehen, dass den Äußerungen ein überwiegender Meinungsäußerungsgehalt zukomme, so dass für die Anwendung von $\S 824$ BGB in der Regel kein Raum sei. In diesem Zusammenhang sei in der Rechtsprechung anerkannt, dass die mit der Veröffentlichung solcher Tests verbundene Meinungsäußerung keinen rechtswidrigen Eingriff darstelle, wenn die Untersuchung und Bewertung neutral, objektiv, sachkundig und sorgfältig unter Anwendung einer vertretbaren Bewertungsmethode erfolgt sei. Seien diese Anforderungen der Neutralität, Objektivität und Sachkunde gewahrt, bestehe für den sich Äußernden aufgrund des Art. 5 Abs. 1 GG ein erheblicher Beurteilungsspielraum, der Grenzen nur noch dort erfahre, wo
- die Bewertung nicht mehr sachbezogen sei, sondern reine Schmähkritik darstelle;

- die Bewertung offensichtlich unrichtig ist, es sich um bewusste Fehlurteile oder bewusste Verzerrungen handele oder

- die Bewertung eigenständige, nicht in ihr aufgehende und ihr untergeordnete unrichtige Tatsachenbehauptungen enthalte.

Anhand dieser Kriterien sei vorliegend eine Rechtsverletzung des Pharmaunternehmens nicht gegeben. Insbesondere sei für das Gericht nicht erkennbar, dass die erforderliche Objektivität und Neutralität bei der Erstellung der NVL im hier maßgeblichen Zusammenhang nicht gewahrt worden wäre.

Wettbewerbsrechtliche Unterlassungs- und Berichtigungsansprüche bestehen nach Auffassung des Gerichts nicht, da mit den Äußerungen in der NVL ein „geschäftliches Handeln zu Zwecken des Wettbewerbs“ nicht erkennbar sei. Damit fehle es an einer tatbestandlichen Grundvoraussetzung für die Anwendung von Wettbewerbsrecht. Eine geschäftliche Handlung erfordere zwar keine Wettbewerbsabsicht, setze aber doch einen objektiven Zusammenhang zwischen der Handlung und der Förderung eigenen oder fremden Wettbewerbs voraus. Die Handlung müsse daher bei objektiver Betrachtung darauf gerichtet sein, den Absatz oder Bezug von Waren zu fördern. An einer solchen objektiven Zielbestimmung fehle es vorlie- 
gend. Die beanstandeten Äußerungen könnten sich zwar auf den Wettbewerb auswirken, sie seien aber im Rahmen der definierten Aufgabenstellung der NVL zu gänzlich anderen, nichtgeschäftlichen Zielen erfolgt und dienten daher auch objektiv nicht der Wettbewerbsbeeinflussung.

Mit seiner Entscheidung bestätigt das Landgericht die auch von hiesiger Seite vertretene Auffassung, dass Nationale Versorgungsleitlinien und die Leitlinien der einzelnen medizinisch-wissenschaftlichen Fachgesellschaften wegen ihrer eigentlichen wissenschaftlichen Zielsetzungen einer wettbewerbsrechtlichen Beurteilung entzogen und damit jedenfalls insoweit nicht justiziabel sind.

Dies gilt allerdings nicht für etwaige, vom jeweiligen Anspruchsteller nachzuweisende, unwahre Tatsachengrundlagen in Leitlinien, die zu Rechtsverletzungen der jeweiligen Anspruchsteller führen können. Soweit es dadurch - kausal - zu einer Kreditgefährdung, also einer Schädigung des Umsatzes oder des gewerblichen Fortkommens des jeweiligen Unternehmens kommt, sind auch Schadensersatzansprüche Dritter gegenüber den Trägern der NVL und den einzelnen medizinisch-wissenschaftlichen Fachgesellschaften und den Autoren und Herausgebern der Leitlinien denkbar.
Das Urteil des Landgerichts Köln bekräftigt die Freiheit von medizinischer Wissenschaft und Forschung und den ungehinderten, unabhängigen wissenschaftlichen Diskurs unter den Beteiligten. Es trägt damit den durch das Grundgesetz geschützten Rechtspositionen der medizinischen Wissenschaften und der Meinungsäußerungsfreiheit in besonderer Weise Rechnung.

(Landgericht Köln, Urteil vom 30.11.2011 - 280 523/11 - nicht rechtskräftig)

\section{Bitte zitieren als}

Wienke A. Medizinische Leitlinien sind wettbewerbsrechtlich nicht justiziabel. GMS Mitt AWMF. 2011;8:Doc30.

DOI: 10.3205/awmf000246, URN: urn:nbn:de:0183-awmf0002467

Artikel online frei zugänglich unter

http://www.egms.de/en/journals/awmf/2011-8/awmf000246.shtml

Eingereicht: 05.12.2011

Veröffentlicht: 06.12.2011

\section{Copyright}

(C)2011 Wienke. Dieser Artikel ist ein Open Access-Artikel und steht unter den Creative Commons Lizenzbedingungen

(http://creativecommons.org/licenses/by-nc-nd/3.0/deed.de). Er darf vervielfältigt, verbreitet und öffentlich zugänglich gemacht werden, vorausgesetzt dass Autor und Quelle genannt werden. 